\title{
place de l'odontologiste dans la prise en charge pluridisciplinaire d'un patient âgé dénutri
}

RÉSUMÉ L'altération de l'état nutritionnel chez la personne âgée a des répercussions importantes en termes de morbidité et de mortalité. La dénutrition protéino-énergétique, dont le diagnostic repose sur des indices anthropométriques, biologiques et diététiques clairement définis, affecte majoritairement les sujets institutionnalisés. Cependant, elle atteint également les personnes âgées vivant à domicile, en particulier lorsqu'elles souffrent de dépendance ou d'isolement social. Les pathologies bucco-dentaires peuvent constituer des facteurs de risque d'apparition d'un état de dénutrition et il convient de les prendre en charge. La priorité est l'as-

\section{A. BODINEAU-MOBARAK} Service d'Odontologie, Hôpital Louis Mourier, Colombes AP-HP.

\section{A. GIACOBBI \\ UFR Odontologie, Paris 5.}

\section{J.-C. TAVERNIER \\ UFR Odontologie,}

Paris 5.

\section{Marysette FOLLIGUET} MCU-PH,

Chef du Service d'Odontologie,

Service d'Odontologie,

Hôpital Louis Mourier,

Colombes AP-HP.

\section{MOTS CLÉS}

dénutrition

personnes âgées

facteurs bucco-dentaires 


\section{introduction}

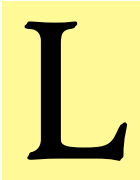

e statut nutritionnel est un déterminant pronostique de l'état de santé et de la survie chez la personne âgée. La dénutrition protéino-énergétique est source d'infections, de fonte musculaire, de diminution des capacités fonctionnelles et d'immobilisme et son lien avec l'augmentation de la morbidité et de la mortalité est clairement établi. Elle multiplie par 2 à 6 la morbidité infectieuse et par 2 à 4 la mortalité. Or, la prévalence de la dénutrition, évaluée à $5 \%$ dans la population des personnes âgées de plus de 65 ans vivant à leur domicile, atteint $50 \%$ à l'entrée dans un service hospitalier de court séjour[1] et se situe, selon les auteurs, entre 20 et
$70 \%$ chez les patients institutionnalisés en unités de soins de longue durée[2].

L'apparition d'une dénutrition chez le sujet âgé est généralement multifactorielle et dépend de l'état de santé général de la personne, de son statut socioéconomique, de ses habitudes alimentaires et de son état dentaire. C'est pourquoi il est souvent difficile d'établir un lien direct entre dénutrition et pathologies buccales. Cependant, il a été observé des déficits en fer, en vitamine $A$, vitamine $C$, acide folique, thiamine et en protéines, ainsi qu'un risque accru de morbidité et mortalité, chez les sujets âgés présentant un état dentaire défectueux[3].

\section{critères diagnostiques de la dénutrition}

Le diagnostic d'un état de malnutrition ou de dénutrition est réalisé à l'aide d'indices anthropométriques, biologiques et diététiques (voir tableau I). Ils sont à analyser les uns avec les autres pour avoir un bilan nutritionnel précis (gravité de la dénutrition, causes, conséquences biologiques). L'association de certains de ces indices a permis le développement d'indices globaux, particulièrement utilisés en gériatrie.

\section{les indices anthropométriques}

La perte de poids non volontaire est le premier reflet d'un état de dénutrition protéino-énergétique chez la personne âgée. Il est donc indispensable que des pesées soient effectuées régulièrement, de préférence avec l'aide d'un soignant, afin d'avoir une courbe de poids précise.

L'Indice de Masse Corporelle (IMC) ou Body Mass Index (BMI) est un bon marqueur de la corpulence de la personne. La difficulté principale du calcul de l'IMC chez la personne âgée réside dans le fait qu'il n'est souvent pas aisé d'estimer la taille réelle du sujet. Il est donc généralement admis de calculer la taille à partir de la mesure de la distance talon-genou qui reste fixe avec l'âge.

D'autres indices anthropométriques sont utilisés, bien que plus rarement. Il 
Tableau I

Critères d'évaluation du statut nutritionnel chez la personne âgée

\begin{tabular}{|c|c|c|c|}
\hline Indices anthropométriques & Indices biologiques & $\begin{array}{l}\text { Indices } \\
\text { diététiques }\end{array}$ & Indices globaux \\
\hline $\begin{array}{c}\text { Perte de poids } \\
\text { dénutrition } \mathrm{si} \geq 2 \mathrm{~kg} \text { en } 1 \text { mois } \\
\text { ou } \geq \text { à } 10 \% \text { du poids en } 6 \text { mois }\end{array}$ & $\begin{array}{c}\text { Albuminémie } \\
>\text { satisfaisant si } 35<\text { alb }<50 \mathrm{~g} / \mathrm{l} \\
>\text { dénutrition modérée } \\
\text { si } 30<\text { alb }<35 \mathrm{~g} / \mathrm{l} \\
>\text { dénutrition sévère } \\
\text { si alb } \leq 30 \mathrm{~g} / \mathrm{l} \\
>\text { dénutrition grave } \\
\text { si alb } \leq 25 \mathrm{~g} / \mathrm{l}\end{array}$ & $\begin{array}{l}\text { Enquêtes } \\
\text { alimentaires }\end{array}$ & $\begin{array}{c}\text { Geriatric Nutritional Risk Index } \\
\text { (GNRI) } \\
\text { > satisfaisant si GNRI > } 100 \\
>\text { à risque de dénutrition } \\
\text { si } 100>\text { GNRI > 97,5 } \\
\text { > dénutrition si GNRI < } 97,5\end{array}$ \\
\hline $\begin{array}{c}\text { Indice de Masse Corporelle (IMC) } \\
=\text { Body Mass Index (BMI) } \\
=\text { poids }(\mathbf{k g}) / \text { taille }(\mathbf{m} \mathbf{2}) \\
\text { dénutrition si IMC } \leq 21 \mathrm{~kg} / \mathrm{m} 2 \\
\text { chez l'homme } \\
\text { et } \leq 19 \mathrm{~kg} / \mathrm{m} 2 \text { chez la femme }\end{array}$ & $\begin{array}{l}\text { Transthyrétinémie } \\
=\text { pré-albuminémie } \\
\text { risque si }<200 \mathrm{mg} / \mathrm{l}\end{array}$ & $\begin{array}{l}\text { Mesure des } \\
\text { Ingesta }\end{array}$ & $\begin{array}{c}\text { Mini Nutritional Assessment } \\
\text { (MNA) } \\
>\text { satisfaisant si MNA }>23 \\
>\text { à risque de dénutrition } \\
\text { si } 23>\text { MNA }>17 \\
>\text { dénutrition si MNA }<17\end{array}$ \\
\hline $\begin{array}{l}\text { Circonférence brachiale } \\
\text { ou du mollet }\end{array}$ & $\begin{array}{l}\text { Protéines de l'inflammation } \\
>\text { protéine C réactive }=\mathrm{CRP} \\
\text { seuil pathologique }>10 \mathrm{mg} / \mathrm{l} \\
\quad>\text { orosomucoïde } \\
\text { seuil pathologique }>1,2 \mathrm{~g} / \mathrm{l}\end{array}$ & & \\
\hline \multirow{2}{*}{ Mesure des plis cutanés } & $\begin{array}{l}\text { État d'hydratation } \\
\text { natrémie et protidémie }\end{array}$ & & \\
\hline & $\begin{array}{l}\text { Numération formule sanguine } \\
\text { lymphopénie }\end{array}$ & & \\
\hline
\end{tabular}

s'agit par exemple de la mesure de la circonférence brachiale ou du mollet permettant d'obtenir une estimation de la masse musculaire ; en effet, la dénutrition est souvent associée à un hypercatabolisme se traduisant par une fonte musculaire. À cet égard, il peut être intéressant de noter l'évolution de la capacité fonctionnelle de la personne âgée, c'est-à-dire une éventuelle altération de ses capacités à se lever et à rester en position debout. De même, la mesure des plis cutanés permet d'estimer la masse grasse, reflet des réserves énergétiques de l'organisme. Toutes ces mesures sont comparées à des normes établies en fonction de l'âge et du sexe de l'individu.

\section{- les indices biologiques}

L'évaluation du taux d'albumine sérique (protéine synthétisée par le foie) permet d'évaluer une dénutrition ou un risque nutritionnel et reste l'élément de référence de l'évolution à long terme de l'état nutritionnel du fait de sa longue demi-vie (21 jours). 
La transthyrétine (ou pré-albumine), également d'origine hépatique, est une protéine de transport des hormones thyroïdiennes. C'est un marqueur très sensible et précoce de la dénutrition protéino-énergétique $d u$ fait de sa demi-vie très courte (48 heures).

Afin de différencier la dénutrition exogène (relative à un déficit des apports nutritionnels) de la dénutrition endogène (résultant d'une situation d'hypercatabolisme dans un contexte de syndrome inflammatoire), les concentrations plasmatiques des protéines, marqueurs de l'état nutritionnel sont toujours à interpréter en fonction de l'état d'hydratation (natrémie et protidémie) et des variations des protéines de l'inflammation telles que la protéine $\mathrm{C}$ réactive (CRP) et l'orosomucoïde.

$\mathrm{Au}$ cours de la dénutrition, apparaît une diminution du nombre de lymphocytes totaux, associée à une susceptibilité accrue aux infections et une exacerbation des phénomènes inflammatoires. Ainsi, la présence d'infections traînantes peut être révélatrice d'une malnutrition et le bilan biologique pourra inclure, par une numération de formule sanguine, l'évaluation des taux sériques de lymphocytes et de monocytes.

\section{- les indices diététiques}

Ces indices reposent sur les enquêtes alimentaires permettant d'estimer les apports journaliers du sujet âgé. La réalisation de ces enquêtes est relativement aisée dans les services hospitaliers et les institutions et consiste le plus souvent à relever de façon régulière les ingesta (c'est-à-dire la mesure des apports alimentaires quantitatifs et qualitatifs quotidiens). En revanche, le recueil de ces données est beaucoup plus difficile pour les personnes âgées vivant à leur domicile, les résultats des enquêtes alimentaires étant généralement peu fiables et/ou incomplets. Il est néanmoins possible de faire appel à l'entourage ou aux aides à domicile afin d'obtenir un recueil plus exhaustif.

\section{- les indices globaux}

De façon intéressante, l'état nutritionnel peut être évalué par le Geriatric Nutritional Risk Index (GNRI) qui a l'avantage d'associer, à l'aide d'une formule mathématique, un paramètre biologique, l'albumine, et un paramètre anthropométrique, le rapport poids actuel sur poids usuel (calculé en fonction de la taille de l'individu).

De même, le Mini Nutritional Assessment (MNA) est une grille d'évaluation qui associe, à travers 18 items, des indices anthropométriques et diététiques, ainsi que des données subjectives et environnementales.

\section{implications des facteurs bucco-dentaires dans la dénutrition}

Le vieillissement de la cavité buccale et les pathologies qui y sont associées entraînent une diminution du potentiel masticatoire et influence le type d'alimentation de la personne âgée, avec un impact sur ses apports nutritionnels 
qualitatifs et quantitatifs, son confort et son bien-être. En dehors de toute pathologie, l'atrophie physiologique de la muqueuse buccale au cours du vieillissement, responsable d'une vulnérabilité aux traumatismes et à la pression durant la mastication, et la perte de masse musculaire striée liée à l'âge (plus importante chez les édentés) expliquent l'éviction de certains aliments durs et riches en fibres.

En outre, Sullivan[4] met en évidence le rôle joué par les troubles buccaux généraux (halitose, hygiène buccale insuffisante, bouche sèche, incapacité à mastiquer, absence d'occlusion, pathologie temporo-mandibulaire, infection-inflammation, lésions muqueuses, douleurs orales) dans la perte de poids de plus de $10 \%$.

\section{- nombre de dents}

La conservation d'un minimum de 20 dents semble être nécessaire au maintien d'une fonction masticatoire correcte et d'une bonne nutrition[5]. Ainsi, la perte de dents non compensée serait responsable de modifications du régime alimentaire[6] et pourrait constituer un facteur de risque d'être en sous-poids, $\mathrm{du}$ fait de la perturbation du potentiel et $\mathrm{du}$ temps masticatoires $[2,7,8]$. Le rôle significatif de l'édentement et du port de prothèses défectueuses a été démontré dans la perte de poids supérieure à $4 \%$, des apports faibles en nutriments et de multiples inadéquations diététiques $[2,9,10]$. Or le nombre moyen de dents perdues augmente avec l'âge et chez les personnes de plus de 65 ans, le nombre de dents restantes varie entre 12 et 17 selon les pays[11, 12]. Ainsi, seuls $10 \%$ des personnes âgées de plus de 75 ans ont 21 dents naturelles ou plus, versus $72 \%$ dans l'ensemble de la population adulte.

Les personnes édentées ont habituellement des prises alimentaires quantitativement inférieures aux niveaux recommandés, perturbations associées à des modifications qualitatives $\mathrm{du}$ régime alimentaire conduisant à l'exclusion de certains aliments. Ceci aboutit à un régime pauvre en fibres, vitamines et protéines et riche en hydrates de carbone[6], avec des conséquences sur la santé générale.

\section{- port de prothèses}

Bien que les patients porteurs de prothèses soient nombreux dans la population âgée, le besoin fonctionnel reste important $[8,13,14]$. Aux USA et au Canada, 50 à $70 \%$ des personnes institutionnalisées ne sont pas satisfaites de leurs prothèses[15] qui, lorsqu'elles sont mal adaptées, peuvent être à l'origine de lésions buccales. Nous avons constaté, lors d'études réalisées en 2001 et 2004 dans deux services de soins de longue durée, que seuls $30 \%$ des édentés totaux possédaient des prothèses et parmi eux, $80 \%$ ne les portaient pas.

Par ailleurs, une augmentation significative $d u$ risque de mortalité à long terme a été montrée chez les patients porteurs de prothèses mal adaptées ou les personnes ayant un état dentaire non fonctionnel, comparés aux sujets présentant une denture fonctionnelle. Cela pourrait être attribué aux conséquences sur le plan nutritionnel (choix des aliments, aptitude à mastiquer,...) [7]. 


\section{- pathologie carieuse}

Outre le nombre de dents, l'état dentaire est également à prendre en compte dans la détérioration $d u$ potentiel masticatoire et l'apparition d'une malnutrition. En effet, le vieillissement de l'organe dentaire, associé à un régime alimentaire riche en sucres, à une hygiène buccale défectueuse et à une diminution de la sécrétion salivaire, constituent des facteurs de risque d'apparition de lésions carieuses. Ainsi le risque carieux, souvent élevé dans l'enfance, a tendance à se stabiliser chez l'adulte d'âge moyen pour augmenter de nouveau de façon importante chez la personne âgée. Les lésions atteignant le collet et les racines dentaires constituent une pathologie relativement spécifique du sujet âgé et leur prévalence augmente de 18 à $51 \%$ avec l'âge[13]. En outre, les difficultés que rencontrent les personnes âgées à accéder aux soins bucco-dentaires participent à l'augmentation de cette prévalence et près de $75 \%$ d'entre eux présenteraient de 1 à 4 dents à soigner[16].

Ces différents éléments concourent à favoriser les troubles de la mastication et ont été incriminés dans l'apparition de malnutrition chez le sujet âgé, comme le révèle l'étude de Yoshihara et al.[17] qui associe la diminution du taux d'albumine à la fréquence des caries radiculaires.

\section{- hyposialie}

La diminution de la sécrétion salivaire, particulièrement fréquente dans la population âgée, occupe une place

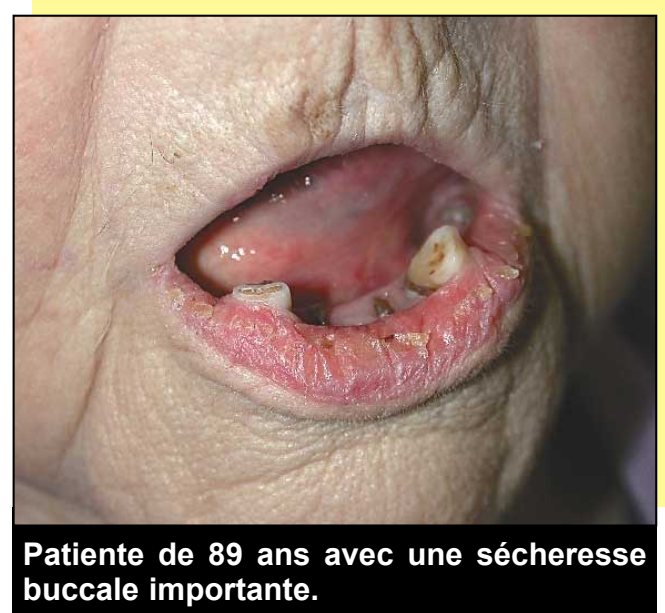

prépondérante parmi les causes de modification $d u$ régime alimentaire. En effet, la salive a un rôle important de lubrifiant et de protection de la cavité buccale. En conséquence, l'hyposialie est à l'origine de difficultés à s'alimenter, tout particulièrement en ce qui concerne les aliments durs et/ou secs, et entraîne des perturbations dans la constitution du bol alimentaire susceptibles de compromettre le statut nutritionnel[9]. De plus, elle augmente considérablement le risque d'apparition de caries radiculaires et rend difficile le port de prothèses amovibles. Il a été mis en évidence un lien statistique entre la réduction forte des flux salivaires, stimulés ou non, et la dénutrition sévère $[18,19]$ ou le taux d'albumine[20]. Il est important de souligner que l'état de dénutrition s'accompagne fréquemment d'une déshydratation et que cette dernière peut être à l'origine de l'apparition d'un syndrome sec buccal ou aggraver une hyposialie préexistante. 
- pathologies parodontales

La diminution de la dextérité manuelle et la perte d'autonomie représentent des obstacles majeurs à la réalisation des soins d'hygiène buccale et favorisent l'accumulation de plaques bactériennes et le développement de maladies parodontales, retrouvées chez 55 à $85 \%$ des personnes âgées[13,14]. La pathologie est alors fréquemment associée à des douleurs et des mobilités dentaires susceptibles de perturber la fonction masticatoire et l'inflammation gingivale évoluant sur un mode chronique peut participer à l'altération des réserves énergétiques de sujets déjà fragiles. Ainsi, il a été montré que l'Indice de Masse Corporelle (IMC) est plus faible chez les sujets atteints de parodontite sévère[21]. En outre, les altérations des réponses immunitaires liées à la dénutrition entraînent des difficultés de cicatrisation et une moindre résistance aux agents pathogènes.

\section{co-morbidités}

Enfin, il semble que certaines co-morbidités, fréquentes chez le sujet âgé, contribuent par elles-mêmes à altérer la fonction masticatoire. Il en est ainsi, par exemple, de la maladie de Horton qui peut entraîner une claudication intermittente des muscles masticateurs et de la langue, des paralysies faciales consécutives d'accidents vasculaires cérébraux, de la maladie de Parkinson et la maladie d'Alzheimer associant des troubles de la mastication et de la déglutition. Il semble également que ces deux dernières pathologies, tout comme le diabète, puissent favoriser l'apparition d'une hyposialie et d'un syndrome sec buccal[15, 22], indépendamment des thérapeutiques psychotropes qui leur sont souvent associées.

\section{conduite à tenir pour l'odontologiste devant un patient âgé dénutri ou à risque de dénutrition}

La population âgée n'est pas homogène et tous les sujets qui la composent ne présentent pas le même risque de malnutrition et/ou dénutrition. Il est donc nécessaire de distinguer les différentes situations auxquelles l'odontologiste peut être confronté afin de répondre au mieux à chacune d'elles. Cependant, nous n'aborderons pas le cas des sujets présentant une dénutrition sévère qui met en jeu le pronostic vital, puisque ces situations requièrent une hospitalisation, parfois associée à une renutrition parentérale ou entérale par sonde naso-gastrique.

Les personnes âgées vivant en institution ou en service hospitalier de soins de longue durée sont les plus exposées au risque de dénutrition, du fait de leur état de santé précaire et de leur polypathologie qui s'accompagne de handicap et de dépendance. Cepen- 
dant, cette fraction ne représente que $10 \%$ de la population âgée et est généralement bien suivie sur le plan médical, en particulier nutritionnel. S'il est peu fréquent que le chirurgien dentiste omnipraticien prenne en charge ces patients, il n'en demeure pas moins que leur statut nutritionnel est généralement connu. La prise en charge buccodentaire privilégiera d'abord l'assainissement de la cavité buccale, la suppression des foyers infectieux et des états inflammatoires (qui fragilisent les réserves énergétiques de l'individu) et de tout ce qui peut être source de douleur et d'inconfort. Il n'est pas rare de préconiser la suppression du port des prothèses amovibles existantes, qui sont souvent anciennes, inadaptées, à l'origine de blessures ou de candidose,... Une réhabilitation prothétique peut être proposée dans un second temps, mais ne sera envisagée que si le patient le désire réellement et si ses capacités physiques et cognitives lui permettent d'adhérer et de participer à la démarche thérapeutique. La réalisation de prothèses amovibles, en réponse à une demande de l'entourage, est vouée à l'échec puisque, même si les prothèses sont une réussite par ellesmêmes, elles ne seront jamais portées. L'expérience montre que l'assainissement buccal seul, malgré la présence d'édentements non compensés, permet l'amélioration du confort et le retour à des prises alimentaires jugées satisfaisantes. Ceci n'exclut pas que la prise en charge globale du problème nutritionnel puisse inclure la prescription de compléments nutritionnels protéino-énergétiques.

Les personnes âgées vivant à leur domicile représentent près de $90 \%$ des plus de 65 ans et, là encore, cette popu-

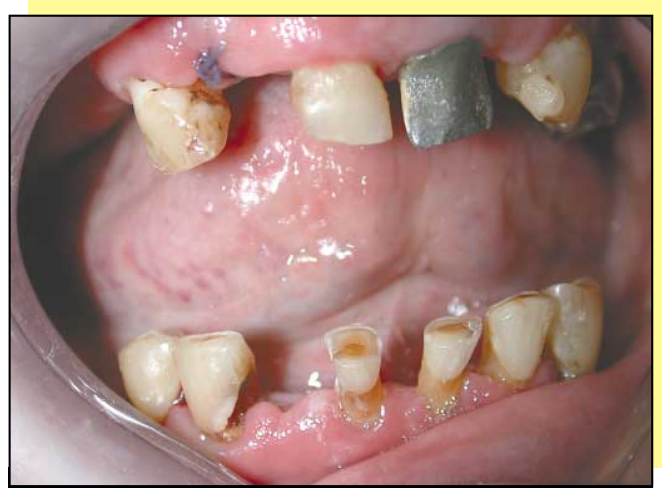

État bucco-dentaire d'une patiente de 92 ans.

lation est hétérogène. En effet, il est classique de distinguer trois catégories de sujets âgés : $70 \%$ sont en bonne santé et ne présentent qu'un faible risque de malnutrition/dénutrition. Il est cependant nécessaire d'être vigilant dans les cas de personnes vivant seules, la solitude étant un facteur de risque important de malnutrition. Une deuxième catégorie (20\% des sujets âgés à domicile) est constituée de personnes «en voie de fragilisation», c'est-à-dire présentant une ou plusieurs pathologies aiguës ou chroniques qui les fragilisent et qui sont susceptibles de les carencer sur le plan nutritionnel. Enfin, la troisième catégorie est constituée de personnes malades dépendantes qui, malgré les aides à domicile et le portage des repas, sont souvent en situation de carences nutritionnelles. C'est au sein de ces deux dernières populations (30\% des personnes âgées à domicile) que l'on trouve la majorité des situations où tout évènement intercurrent provoque l'hospitalisation en urgence pour la décompensation d'un état fragile au préalable.

L'omnipraticien est susceptible de prendre en charge au cabinet ces per- 
sonnes vivant à leur domicile et, en tant que professionnel de la cavité buccale, doit avoir une démarche de prévention et de dépistage des pathologies nutritionnelles. Ceci passe tout d'abord par des questions simples sur les habitudes alimentaires : la préparation, la fréquence et la régularité des repas, l'évolution du poids au cours des derniers mois, le bien-être buccal, le ressenti de l'efficacité masticatoire,... Des prothèses amovibles inadaptées, décrites comme «trop grandes» par le patient, peuvent être révélatrices d'une fonte osseuse et musculaire qui doit alerter. Il est également indispensable de faire le bilan des pathologies générales du patient et des traitements en cours, en gardant à l'esprit que la polymédication, les régimes sans sel et les régimes diabétiques sont anorexigènes. Une vigilance toute particulière doit être portée aux patients fragiles qui peuvent présenter une dénutrition modérée non diagnostiquée; il est alors souvent nécessaire de contacter le médecin traitant afin d'instaurer une prise en charge globale pouvant inclure des soins bucco-dentaires mais également la prescription de compléments nutritionnels ou d'une aide à domicile. Des conseils diététiques peuvent être donnés (voir tableau II) en recommandant le fractionnement des repas (notamment une collation en milieu d'aprèsmidi) et une légère activité physique (la marche par exemple).

Tableau II

Recommandations nutritionnelles chez la personne âgée

\begin{tabular}{|c|c|c|}
\hline & Rôles & Apports nutritionnels conseillés \\
\hline Protéines & $\begin{array}{l}>\text { constituants fondamentaux des tissus } \\
\text { rôles structurel, enzymatique, immunologi- } \\
\text { que et métabolique }\end{array}$ & $\begin{array}{c}10 \text { à } 15 \% \text { de la ration calorique journalière } \\
\text { (protéines animales et végétales) } \\
\text { soit } 1 \text { à } 1,5 \mathrm{~g} / \mathrm{kg} / \mathrm{j}\end{array}$ \\
\hline Lipides & $\begin{array}{l}>\text { rôle structurel (membranes cellulaires) } \\
>\text { rôle fonctionnel (transmission intra-cellulaire) }\end{array}$ & $\begin{array}{l}30 \text { à } 35 \% \text { de la ration } \\
\text { calorique journalière }\end{array}$ \\
\hline Glucides & $\begin{array}{l}>\text { rôle énergétique de courte durée } \\
>\text { activités physiques et intellectuelles }\end{array}$ & $\begin{array}{c}\mathbf{5 0} \text { à } \mathbf{5 5} \% \text { de la ration calorique journalière } \\
>\text { glucides simples } \\
\text { (saccharose, fructose, glucose) } \\
>\text { glucides complexes } \\
\text { (pain, riz, pâtes, pommes de terre, céréales) }\end{array}$ \\
\hline Fibres & $\begin{array}{l}>\text { peu ou pas énergétiques } \\
>\text { rôle satiétogène } \\
>\text { induction de la contraction des muscles intes- } \\
\quad \text { tinaux } \\
>\text { limitation de l'hyperglycémie postprandiale } \\
>\text { effet hypocholestérolémiant }\end{array}$ & 25 à $30 \mathrm{~g} / \mathrm{j}$ \\
\hline Apports hydriques & & 1,5 à 2 litres d'eau / jour \\
\hline $\begin{array}{l}\text { Micronutriments } \\
\text { (vitamines et } \\
\text { minéraux) }\end{array}$ & nombreuses fonctions biologiques & $\begin{array}{c}\text { apportés en quantité suffisante } \\
\text { par une alimentation } \\
\text { diversifiée }\end{array}$ \\
\hline
\end{tabular}


En ce qui concerne la prise en charge bucco-dentaire :

- supprimer les foyers infectieux et états inflammatoires qui favorisent l'hypercatabolisme et fragilisent les réserves énergétiques du patient ;

- prendre en charge toute cause de douleur qui compromet l'alimentation ;

- porter une vigilance particulière lors de certains traitements (tels que les extractions) qui peuvent aggraver de façon transitoire les douleurs et l'inconfort buccal, et donc rendre encore plus difficile les prises alimentaires ;

- tenir compte du fait que la dénutrition favorise l'immunodépression et les pathologies infectieuses et que les temps de cicatrisation sont souvent allongés ;
- favoriser les traitements simples et réalisables rapidement, qui sont souvent les plus appropriés chez ces patients déjà fragiles et fatigués ;

- chez les patients présentant une pathologie chronique dont on sait qu'elle va s'aggraver (ex: démence débutante), mettre en place des mesures préventives et réaliser l'assainissement de la cavité buccale avant que leur état ne se détériore.

Enfin, la texture des aliments a un rôle primordial dans le plaisir de consommer une alimentation variée ; c'est pourquoi il est recommandé de restaurer une fonction masticatoire satisfaisante, via une réhabilitation prothétique, en adaptant les choix thérapeutiques aux capacités physiques et cognitives de chaque patient.

\section{conclusion}

Le dépistage d'une malnutrition ou d'une dénutrition doit être systématique lors de la consultation odontologique d'une personne âgée, quel que soit son état de santé. Ce dépistage peut être réalisé à l'aide de questions simples concernant la diversité des aliments consommés, la fréquence des repas, le confort buccal, le ressenti de l'efficacité masticatoire, l'évolution du poids corporel... Lorsqu'une altération du statut nutritionnel est suspectée, il est nécessaire d'envisager, en collaboration avec le médecin traitant, la réalisation d'un bilan nutritionnel complet. L'assainissement de la cavité buccale et la restauration de la fonction masticatoire participent à la prise en charge globale de la dénutrition ; il faut privilégier les solutions thérapeutiques simples et réalisables rapidement.

\section{bibliographie}

1. Constans $T$, Lesourd $B$, Alix E, Dardaine V.

Alimentation

et état nutritionnel des personnes âgées en France : domicile et hôpital. Prévenir 1994;26:93-99.
2. Marcenes W, Steele JG, Sheiham A, Walls AW. The relationship between dental status, 
food selection, nutrient intake, nutritional status, and body mass index in older people. Cad Saude Publica 2003; 19(3):809-816.

3. Papas AS, Palmer CA Rounds MC, Russell RM. The effects of denture status on nutrition. Spec Care Dent 1998;18(1): 17-25.

4. Sullivan $\mathrm{DH}$, Martin W, Flaxman N, Hagen JE. Oral health problems and involuntary weight loss in a population of frail elderly. J Am Geriatr Soc 1993; 41(7):725-731.

5. Steele JG, Sheiham A, Marcenes W, Fay N, Walls AW.

Clinical and behavioural risk indicators for root caries in older people. Gerodontology 2001;18(2): 95-101.

6. Pajukoski $\mathrm{H}$, Meurman JH, Halonen P, Sulkava R. Prevalence of subjective dry mouth and burning mouth in hospitalized elderly patients and outpatients in relation to saliva, medication and systemic diseases. Oral Surg Oral Med Oral Pathol Oral Radiol Endod 2001;92(6):641-649.

7. Appollonio I, Carabellese C, Frattola A, Trabucchi M. Influence of dental status on dietary intake and survival in community-dwelling elderly subjects. Age \& Ageing 1997;26: 445-456.

8. Liedberg $B$, Norlén $P$, Öwall B, Stoltze K. Masticatory and nutritional aspects on fixed and removable partial dentures. Clin Oral Invest 2004;8: 11-17.
9. Nagler RM.

Salivary glands and the aging process: mechanistic aspects, health-status and medicinal-efficacy monitoring. Biogerontology 2004;5: 223-233.

10. Ritchie CS, Joshipura $K$, Silliman RA, Miller B, Douglas CW. Oral health problems and significant weight loss among communitydwelling older adults. J Gerontol A Biol Sci Med Sci 2000 I;55(7):M366-371.

11. Fure S, Zickert I. Incidence of tooth loss and dental caries in 60-, 70- and 80-year-old Swedish individuals. Community Dent Oral Epidemiol 1997;25:137-142.

12. Hamalainen $\mathrm{P}$, Meurman $\mathrm{JH}$ Keskinen M, Heikkinen E. Changes in dental status over 10 years in 80-yearold people: a prospective cohort study.

Community Dent Oral Epidemiol 2004; 32:374-384.

13. Bailey RL, Ledikwe JH, Smiciklas-Wright $\mathrm{H}$, Mitchell DC, Jensen GL. Persistent oral health problems associated with comorbidity and impaired diet quality in older adults. J Am Diet Assoc 2004; 104:1273-1276

14. Joly JP, Deville de Perière $D$, Delestan C.

L'accès aux traitements bucco-dentaires chez les personnes âgées dépendantes hospitalisées.

Rev Gériatrie 2000;25(8): 547-552.

15. Ghezzi EM, Ship JA. Systemic diseases and their treatments in the elderly: impact on oral health. J Public Health Dent 2000; 60:289-296.
16. Mack $F$, et al.

Caries and periodontal disease of the elderly in Pomerania, Germany: results of the study of health in Pomerania. Gerodontology 2004;21: 27-36.

17. Yoshihara A, Hanada N, Miyazaki $\mathrm{H}$.

Association between serum albumin and root caries in communitydwelling older adults. J Dent Res 2003;82: 218-222.

18. Mojon P, Budtz-Jorgensen $E$, Michel JP.

Oral health and history of respiratory tract infections in frail institutionalized elders. Gerodontology 1997;14: 9-16.

19. Narhi TO, Vehkalahti MM, Siukosaari P, Ainamo A. Salivary findings, daily medication and root caries in the old elderly. Caries Res 1998;32(1):5-9.

20. Dormenval $\mathrm{V}$, Budtz-Jorgensen $E$, Mojon $P$, Bruyère $A$, Rapin $\mathrm{CH}$.

Associations between malnutrition, poor general health and oral dryness in hospitalized elderly patients.

Age \& Ageing 1998;27: 123-128.

21. Saremi $A$ et al. Periodontal disease and mortality in type 2 diabetes.

Diabetes Care 2005;28: 27-32.

22. Nordenram G, Ryd-Kjellen $E$, Johansson G, Nordstrom G Winblad B.

Alzheimer's disease, oral function and nutritional status. Gerodontology 1996;13: 9-16. 


\section{SUMMARY}

Role of the dental practitioner in the multidisciplinary management of the malnourished elderly patient

A. BODINEAU-MOBARAK, A. GIACOBBI, J.-C. TAVERNIER, M. FOLLIGUET

The deterioration of the nutritional state in the elderly person has important repercussions in terms of morbidity and mortality. Nutrition deficient in high-calorie proteins, for which the diagnosis is based on the Body Mass Index, with biological and dietary factors clearly defined, affects predominantly people in institutions. However, it also strikes elderly people living at home, particularly when they are

keywords: malnutrition, elderly, oral status. infirm or socially isolated. Oral-dental pathologies can constitute risk factors for the onset of malnutrition and they should be taken care of. Priority should be given to the care of the oral cavity in order to encourage nourishment. Prosthetic restoration can be considered secondarily, in adapting the therapeutic possibilities to the physical and cognitive capacity of each patient.

\section{Questions-réponses}

1/ Une perte de poids non volontaire de plus de $10 \%$ du poids en 6 mois, est un signe de dénutrition chez la personne âgée
Vrai
Faux

!eג^ : әsuodə̧y

2/ Une albuminémie de $40 \mathrm{~g} / \mathrm{l}$ est un signe de dénutrition chez la personne âgée

Vrai $\square \quad$ Faux

xnef : əsuodə̧y

3/ Un état de dénutrition favorise l'immunodépression et les pathologies infectieuses
Vrai
Faux

!eı^ : әsuodə̧⿻ 